\title{
Does response interference depend on the subjective visibility of flanker distractors?
}

\author{
Brian Maniscalco • Joo Won Bang • Laila Iravani • \\ Franc Camps-Febrer • Hakwan Lau
}

Published online: 4 April 2012

(C) Psychonomic Society, Inc. 2012

\begin{abstract}
Response interference (or response conflict) refers to the phenomenon whereby response times to a target stimulus are longer in the presence of distractor stimuli that indicate contrary motor responses. Response interference has been observed even when the distractor stimuli cannot be discriminated above chance levels. These results raise the question of whether response interference might be driven automatically by the physical distractor stimuli, independently of one's subjective perception of the distractors. Using a modified version of the Eriksen flanker task, we applied metacontrast masks to the flanker stimuli and measured their subjective visibility after each trial. We found converging lines of evidence that the subjective perception of flankers contributed to response interference, over and above the contribution of automatic processing of the stimulus itself. A factorial analysis revealed that the objective, physical congruency of target and flankers and the
\end{abstract}

\footnotetext{
B. Maniscalco $(\varangle) \cdot$ H. Lau

Department of Psychology, Columbia University,

1190 Amsterdam Ave., MC 5501,

New York, NY 10027, USA

e-mail: brian@psych.columbia.edu

J. W. Bang

New York University,

New York, NY, USA

L. Iravani

Bard College,

Annandale-on-Hudson, NY, USA

F. Camps-Febrer

City University of New York,

New York, NY, USA

H. Lau

Donders Institute of Brain, Cognition and Behavior,

Radboud University,

Nijmegen, The Netherlands
}

subjective, perceptual congruency of target and flankers make additive, noninteracting contributions to target response interference, suggesting that the two interference effects originate from independent levels or stages of cognitive processing.

Keywords Cognitive control and automaticity · Visual awareness $\cdot$ Stimulus control

The Eriksen flanker task (Eriksen \& Schultz 1979; Stins, Polderman, Boomsma, \& de Geus, 2007) is a standard behavioral paradigm for studying response interference and cognitive control. In one standard version of the task, participants respond to a target stimulus (e.g., an arrow) in the middle of the screen. The central target stimulus is surrounded by adjacent stimuli ("flankers"). The flanker stimuli can either indicate the same motor response as the central stimulus (congruent), or they can indicate the opposite response (incongruent). The task is designed to induce response interference when the flankers are incongruent with the target. Typically, under stimulus incongruency, target identification is slower and more error prone. This kind of response inference is also thought to induce conflict signals in the brain, specifically in the prefrontal cortex, including the dorsolateral prefrontal and anterior cingulate cortex (Botvinick, Cohen, \& Carter, 2004).

Intuitively, one might think that response interference under target-flanker incongruency is due to the fact that the flankers are perceived to be incongruent with the central target stimulus. However, interestingly, Schwarz and Mecklinger (1995) found that even when the flankers cannot be discriminated at above-chance levels due to backward masking, incongruency between the flankers and the target stimulus can still lead to response interference.

This is in line with research in motor priming (Eimer \& Schlaghecken, 2003; Kouider \& Dehaene, 2007; Neumann 
\& Klotz, 1994; Sumner \& Husain, 2008; Van den Bussche, Van den Noortgate, \& Reynvoet, 2009), in which participants give a motor response to a target stimulus, which is often preceded by a brief presentation of a congruent or an incongruent stimulus (the "prime"). Again, the standard finding is that participants are typically slower and less accurate when the prime is incongruent with the target stimulus. However, it has been shown that even when the prime is not discriminable, stimulus incongruency can still have an effect (Eimer \& Schlaghecken, 2002; Kouider \& Dehaene, 2007; Neumann \& Klotz, 1994; Sumner \& Husain, 2008). In Vorberg, Mattler, Heinecke, Schmidt, and Schwarzbach's (2003) experiment, it was even shown that the magnitude of the priming effect did not depend on the discriminability of the prime.

These results seem to afford two different interpretations. One possible account is that response interference does not depend on the manner in which distractor stimuli are subjectively perceived, but only on automatic processing of the distractors' physical identity. On this account, the physical incongruency of the target and distractor stimuli by itself would be sufficient to generate response interference, in a somewhat automatic fashion, independently of the observer's subjective experience of the distractors.

However, an alternative account is that even though subjective perception of the distractor identities is not necessary for response interference to occur, it nonetheless contributes to response interference. That is, stimulus incongruency may be able to induce response interference on its own, but the subjective perception of incongruency may further strengthen this effect. This alternative account speaks to the potential contributions of perceptual awareness to cognitive functioning (Lau, 2009; Merikle, Joordens, \& Stolz, 1995).

The second account may seem supported by the results of Schwarz and Mecklinger (1995), mentioned earlier. In that study, the authors conducted an Eriksen flanker task in which the flanker processing was disrupted by backward masks presented at variable stimulus onset asynchronies (SOAs). They found that when flankers were masked with longer SOAs, participants were more able to perceive the identity of the flankers. Although response interference effects occurred even at an SOA at which the average flanker discrimination performance was at chance, the size of the response interference effect increased as SOA (and thus flanker discriminability) increased. This seems to suggest that the subjective perception of distractor stimuli may contribute to response interference effects. However, one problem with this interpretation is that in Schwarz and Mecklinger's study, both the physical flanker stimulus and the level of subjective flanker perception changed together as SOA changed. So, it is possible that the response interference effect was actually being driven by the physical properties of the flanker stimuli-specifically, the physical stimulus strength of the flankers as determined by SOArather than by subjective flanker perception per se.

To resolve this issue, we would need to examine how response interference is affected separately by the objective, physical identity of distractor stimuli versus the subjective, perceived identity of the distractors. One way of doing so would be to collect stimulus classification judgments and subjective visibility ratings for the distractor (flanker) stimuli in every trial. At near-threshold levels of stimulus presentation, perceptual judgments and visibility ratings fluctuate even when the stimulus is kept constant. Thus, by acquiring trialby-trial data on the participants' perceptual judgments regarding the identity and visibility of the flankers, we could test for the contribution of subjective perception to the effect of response interference in the flanker task.

\section{Method}

Participants

A group of 29 students from the Columbia University undergraduate population participated in the experiment. The participants gave informed consent and were paid $\$ 10$ for approximately $1 \mathrm{~h}$ of participation. The research was approved by the Columbia University's Committee for the Protection of Human Subjects.

\section{Materials and Procedure}

The participants were seated in a dimmed room $60 \mathrm{~cm}$ away from a computer monitor. The stimuli were generated using Psychophysics Toolbox (Brainard, 1997; Pelli, 1997) in MATLAB (MathWorks, Natick, MA) and were shown on an iMac monitor (LCD, 24-in. monitor size, 1,920× 1,200 pixel resolution, $60-\mathrm{Hz}$ refresh rate).

On every trial, a central stimulus ("target") was presented at fixation between two adjacent stimuli presented $1.06^{\circ}$ to the left and right ("flankers") for $33 \mathrm{~ms}$ (Fig. 1). The background was gray, targets were black, and flankers were some shade of gray darker than background. (Flanker contrast relative to the background was adjusted for each participant in order to achieve threshold levels of flanker discrimination performance; see below.) The target and flankers could be either squares or diamonds measuring $0.7^{\circ}$ on each side. The left and right flankers always had identical shapes. Target and flanker shapes were counterbalanced across trials such that knowing the target shape gave no information about the flanker shapes, and vice versa.

After presentation of the target and flankers, metacontrast masks were displayed at the locations of the flankers for $50 \mathrm{~ms}$. The metacontrast masks were constructed by drawing an outline around a diamond superimposed over a square. 

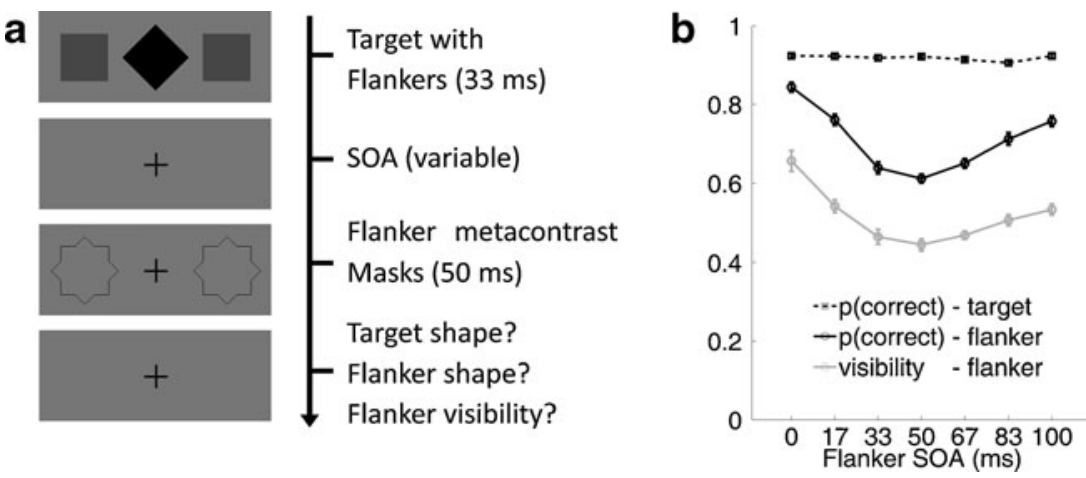

Fig. 1 Task structure and basic task performance. (a) Task: On each trial, a central stimulus (target) was displayed between two adjacent stimuli (flankers). The target and flankers could be squares or diamonds. After a variable stimulus onset asynchrony (SOA), metacontrast masks were displayed at the locations of the flankers. The participant was required to identify the target (square or diamond?) as quickly and accurately as possible. Afterward, the participant identified the flankers and rated their perceived level of visibility on a scale of $1-4$. Flanker contrast was adjusted for each participant in order to achieve threshold levels of

Thus, the masks partially traced the contours of the flankers without physically overlapping them. The time between the onset of the flankers and the onset of the masks (the SOA) varied randomly between trials, in values extending from $0 \mathrm{~ms}$ (i.e., simultaneous onset of the flankers and masks) to $100 \mathrm{~ms}$, in increments of $16.7 \mathrm{~ms}$. SOA was counterbalanced with target/flanker shape pairing.

The participants' task on each trial was, first, to identify the shape of the target as quickly and as accurately as possible. The participants were instructed to identify the shape of the target (square or diamond) as quickly as possible without sacrificing accuracy. Auditory feedback (a lowpitched tone) was presented following incorrect target responses. If no response to the target was provided within $2 \mathrm{~s}$ of the offset of the flanker masks, the text "TOO SLOW!" was displayed in the center of the screen, accompanied by two consecutive low-pitched tones, and the trial was aborted. Target discriminations were entered using the left hand (by pressing the " 1 " and " 2 " keys on the number row of a QWERTY keyboard).

After successfully making the speeded target discrimination, participants made a nonspeeded discrimination of flanker shape (square or diamond). Finally, after making the flanker discrimination, the participants rated the subjective visibility of the flankers. Specifically, they were instructed that they were to judge the subjective appearance of the flankers by rating how clearly they perceived them. The rating was conducted on a scale of 1 (lowest perceived clarity) through 4 (highest perceived clarity). Participants were not provided with specific benchmarks for the meaning of each rating, but rather were encouraged to assign their own meanings to each rating, such that they would use the entire rating scale throughout the experiment. If the flanker discrimination and flanker identification performance. (b) Overall, participants performed the primary task of identifying the central stimulus (target) very well. Identification of the flankers was more difficult. Flanker identification performance and subjective flanker visibility were U-shaped functions of the time between flanker onset and mask onset (the SOA). For presentational purposes, visibility ratings have been scaled to range between 0 and 1 for this graph; in the actual experiment, participants rated subjective flanker visibility on a scale of 1-4. Error bars represent within-subjects standard errors (Morey, 2008)

visibility rating were not completed within $5 \mathrm{~s}$ after the entry of the target discrimination, the trial was aborted. Flanker discriminations and visibility ratings were entered using the right hand (pressing the "7" and " 8 " keys for discrimination, and the "7"-"8"-"9"-"0" keys for visibility rating on the number row of a QWERTY keyboard).

The target and flankers for the next trial were displayed $1 \mathrm{~s}$ after the current trial was terminated, either by successful entry of the flanker visibility rating or by failure to meet the demands of the two response time (RT) limits already mentioned. A crosshair $0.35^{\circ}$ wide was presented at fixation at all times, although it was covered by the target on each trial.

At the start of the experiment, participants completed two practice blocks (50 trials each) and one calibration block (96 trials). In the calibration block, the SOA was fixed at $0 \mathrm{~ms}$. The Weber contrast of the flankers was adjusted continuously between trials using the QUEST threshold estimation procedure (Watson \& Pelli, 1983), with the target level of performance for flanker discrimination set at $84 \%$ correct. Three independent estimates were acquired, with 32 randomly ordered trials contributing to each, and the median estimate of these was used in the main experiment. The main experiment (728 trials) consisted of eight blocks of 91 trials each, with a self-terminated rest period of up to a minute between blocks.

Three participants were omitted from all data analyses because they exhibited chance levels of performance on the flanker discrimination task at all SOAs.

\section{Results}

Overall, the participants performed the main task of identifying the central target with high accuracy $(91.9 \%$ correct), and 
this was not modulated by the flanker-mask SOA $(p=.3)$. Both the accuracy for identification of the flankers and the subjective visibility ratings for the flankers were modulated by flanker-mask SOA $(p s<.001)$, exhibiting significant quadratic trends $(p s<.001)$; specifically, both measures of flanker processing were high at short and long SOAs, with a dip at intermediate SOAs (Fig. 1b). This U-shaped curve obtained by plotting flanker identification performance against SOA is a well-known pattern in metacontrast masking (Breitmeyer \& Öğmen, 2006).

The primary dependent variable of interest in this study was how the RT for target identification depends on the congruency between the target and its flankers. We define stimulus congruency as a match between the objective identities of the target and flankers, as physically displayed on the computer screen. We define perceptual congruency as a match between the perceived identities of the target and flankers, as indicated by participants' trial-by-trial classifications of targets and flankers. We further define the stimulus interference effect as the difference in RTs for target identification between the trials in which the flankers are stimulus-incongruent versus stimulus-congruent - that is, stimulus interference effect $=$ stimulus-incongruent target RT - stimulus-congruent target RT. Likewise, we define the perceptual interference effect thus: perceptual interference effect $=$ perceptually incongruent target RT - perceptually congruent target RT. All analyses of

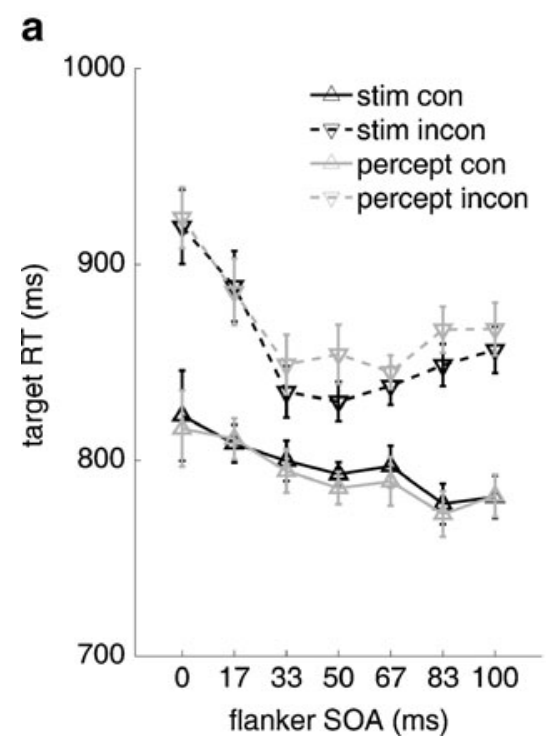

Fig. 2 Response times (RTs) for targets and flankers. (a) Target RTs by target-flanker congruency. Overall, participants were quicker to respond to the target when it had the same shape as the flankers (i.e., when the target and flankers were stimulus-congruent) than when the shapes were different (i.e., when they were stimulus-incongruent). A similar pattern is evident when considering perceptual congruency (i.e., the congruency of the target with the reported flanker identity). (b) Interference effects on target RTs. The effect of target-flanker congruency on target RTs is summarized by plotting curves for the incongruent - congruent target RTs were based on median RTs for those trials on which the target was correctly identified.

Below, we use the general term response interference to refer to flanker-induced interference with target processing (i.e., either the stimulus interference effect or the perceptual interference effect), even though the task design in and of itself does not allow us to ascertain whether the effect of interference occurred at the level of perceptual conflict or of response-level conflict (Morsella et al., 2009). In other words, by "response" interference, we do not mean to imply that the conflict originates at the level of motor responses, only that the effect was measured by means of RTs.

Effect sizes for the stimulus interference effect and perceptual interference effect

In order to assess the effects of stimulus interference and perceptual interference on target processing, we performed a repeated measures analysis of variance (ANOVA) on median target RTs for correct target identifications, with a 2 (congruency type: stimulus/perceptual) $\times 2$ (congruency: congruent/incongruent) $\times 7(\mathrm{SOA})$ design. The data for this analysis are plotted in Fig. 2. (Note that stimulus congruency and perceptual congruency are not fully independent factors here; the stimulus congruency of each trial was defined without regard for its

\section{b}

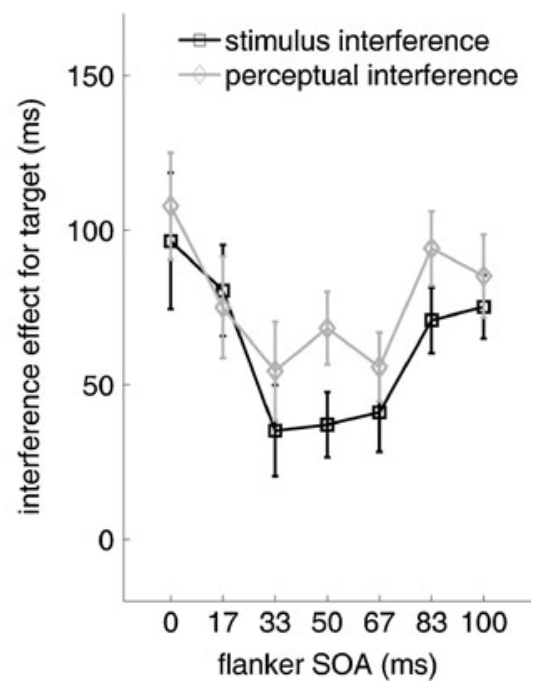

conditions from panel a. Both interference effect curves are U-shaped across SOAs, similarly to the U-shaped functions of flanker identification performance and reported visibility (Fig. 1b). This suggests that flankers interfere more with target processing when the flankers themselves are better processed. Overall, the perceptual interference effect was larger than the stimulus interference effect $(p=.049)$, which suggests that subjective perception of the flankers played a role in response interference over and above that played by the physical stimulus itself. Error bars represent within-subjects standard errors (Morey, 2008) 
perceptual congruency, and vice versa. This limitation is addressed in a subsequent analysis.)

The analysis revealed main effects of congruency $(p<.001)$ and SOA $(p<.001$; quadratic trend, $p=.002)$, demonstrating that target RTs were sensitive to target-flanker congruency and to the strength of flanker processing, as indexed by SOA. These main effects were modulated by a Congruency $\times \mathrm{SOA}$ interaction $(p=.008$; quadratic trend, $p=.007)$. As is shown in Fig. $2 \mathrm{~b}$, this interaction can be interpreted as follows: Both the stimulus interference effect and the perceptual interference effect were U-shaped functions of SOAs, being strongest at short and long SOAs and weakest at intermediate SOAs. Thus, both interference effects were strongest at those SOAs at which the flanker stimuli were more readily discriminable and were rated as more subjectively visible.

Crucially, the analysis also revealed a Congruency Type $\times$ Congruency interaction $(p=.049)$. The source of this interaction was that, overall, the magnitude of the perceptual interference effect $(78 \mathrm{~ms})$ was larger than the magnitude of the stimulus interference effect (64 ms) (Fig. 2b). This finding suggests that the disruptive effects of the flankers on target processing are not attributable only to automatic processing of the stimulus properties, but rather that the manner in which the flankers are subjectively perceived contributes to response interference.

Influence of subjective flanker visibility on the perceptual interference effect

We further probed how subjective flanker perception drives the disruptive effect of the flankers on target processing by considering trial-by-trial subjective reports of flanker visibility. For each participant at each SOA, we classified trials as having "high" or "low" visibility on the basis of a median split. We used a median split rather than considering each visibility rating separately, and performed the median split separately for each SOA, in order to maximize the trial count in each condition. We then performed a repeated measures ANOVA on the median target RTs for correct target identifications, with a 2 (congruency: perceptually congruent/perceptually incongruent) $\times 2$ (reported flanker visibility: high/low $) \times 7(\mathrm{SOA})$ design. Seven subjects were omitted from this analysis because they did not have at least five trials for each Congruency $\times$ Visibility $\times$ SOA data cell. The 19 remaining subjects had an average of 23.5 trials in each of the 28 data cells of the ANOVA analysis. The data for this analysis are plotted in Fig. 3a.

The ANOVA revealed main effects of perceptual congruency $(p=.001)$ and SOA $(p<.001$; quadratic trend, $p=$ .001 ), which were qualified by a Congruency $\times$ SOA interaction $(p=.012$; quadratic $p=.004)$. As in the previous analysis, these results demonstrated a significant effect of perceptual congruency on target RT, and this effect was stronger at those SOAs at which the flanker was more strongly processed.

Crucially, the analysis also revealed a Perceptual Congruency $\times$ Reported Flanker Visibility interaction $(p=$ .049). The source of this interaction was that, overall, the magnitude of the perceptual interference effect was larger when the flankers were reported to be highly visible $(80 \mathrm{~ms})$ than when they were reported to be weakly visible ( $57 \mathrm{~ms}$ ) (Fig. 3b). This finding provides further support for the hypothesis that the disruptive effects of the flankers on target processing partially depend on the subjectively visibility of the flankers.

Because the analysis revealed no interactions involving both reported flanker visibility and SOA ( $p \mathrm{~s}>3)$, we repeated the analysis, this time disregarding the factor of SOA. For each participant, we classified trials as having "high" or "low" visibility on the basis of a median split. We then conducted a repeated measures ANOVA on the median target RTs for correct target identifications, with a 2 (congruency: perceptually congruent/perceptually incongruent) $\times 2$ (reported flanker visibility: high/low) design. This revised analysis allowed us to increase the number of trials in each ANOVA cell and to retain more participants for the analysis. One participant was omitted due to not having at least five trials in each Perceptual Congruency $\times$ Visibility cell. The 25 remaining subjects had an average of 164.4 trials in each of the four data cells of the ANOVA analysis. The data for this analysis are plotted in Fig. 3c.

The ANOVA revealed a main effect of perceptual congruency $(p=.001)$ and a Perceptual Congruency $\times$ Reported Flanker Visibility interaction $(p=.002)$. The source of the interaction was that target RTs were slower when perceptually incongruent trials were highly visible $(870 \mathrm{~ms})$ than when they were weakly visible $(851 \mathrm{~ms})$, whereas target RTs were faster when perceptually congruent trials were highly visible $(791 \mathrm{~ms})$ than when they are weakly visible (808 ms). Thus, the effect of target-flanker perceptual congruency on target RTs in both directions was augmented by flanker visibility.

One concern about these analyses was that reported flanker visibility was correlated with flanker identification performance: When participants rate the flankers as highly visible, they are also more likely to correctly identify those flankers. Thus, the above analyses may have confounded subjective flanker visibility ratings with objective flanker identification performance.

In order to investigate this potential confound, we investigated whether the perceptual interference effect was predicted better by flanker identification performance or flanker visibility ratings. For each participant, we performed two regression analyses. In the first regression, we regressed the perceptual interference effect at each SOA onto $d^{\prime}$ for flanker identification at each SOA. (We used $d^{\prime}$ rather than 


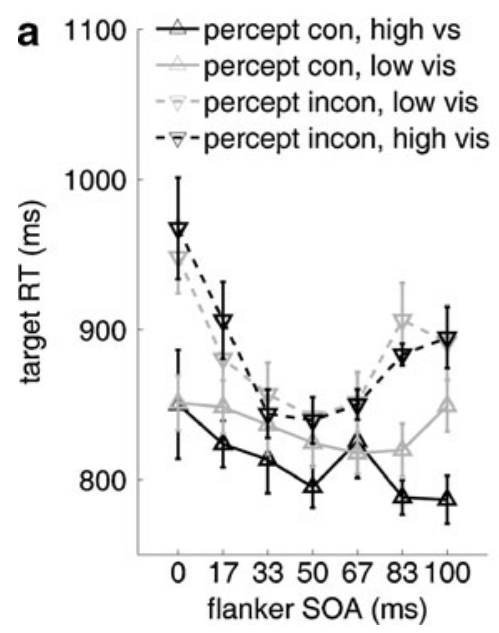

Fig. 3 Perceptual interference effects modulated by subjective flanker visibility. (a, b) On the basis of the trial-by-trial subjective visibility ratings for the flankers, trials were sorted into "high visibility" and "low visibility" bins for each participant at each SOA. The perceptual interference effect (i.e., disruption in target response times caused by a perceived mismatch in target/flanker identities) was larger when participants reported that the flankers were highly visible $(p=.049)$.

percent correct because the former grows linearly with increases in task performance, whereas the latter exhibits progressively smaller increases with increases in task performance, due to ceiling effects (Macmillan \& Creelman, 2005); thus, $d^{\prime}$ is the better choice for a predictor in a linear regression.) In the second regression, we regressed the perceptual interference effect at each SOA onto the average flanker visibility ratings at each SOA. We then compared the mean of the absolute values of the residuals for each participant when using $d^{\prime}$ and average flanker visibility as predictors of the perceptual interference effect with a paired $t$ test.

The average absolute values of the residuals were larger when using $d^{\prime}$ as a predictor $(43.8 \mathrm{~ms})$ than when using average visibility ratings as a predictor $(40.4 \mathrm{~ms})$, numerically consistent with the interpretation that ratings of flanker visibility capture more variance in the perceptual interference effect than does flanker identification performance. However, this difference did not achieve statistical significance $(p=.12)$. Thus, although subjective ratings of flanker visibility modulate the perceptual interference effect, in this analysis it is not clear to what extent this modulation is due specifically to the subjective visibility of the flankers, as opposed to the correlated phenomenon of flanker identification performance.

Independent contributions of stimulus congruency and perceptual congruency to response interference

One caveat to the foregoing analyses is that they do not perfectly discriminate between the effects of stimulus congruency and perceptual congruency. This is because the

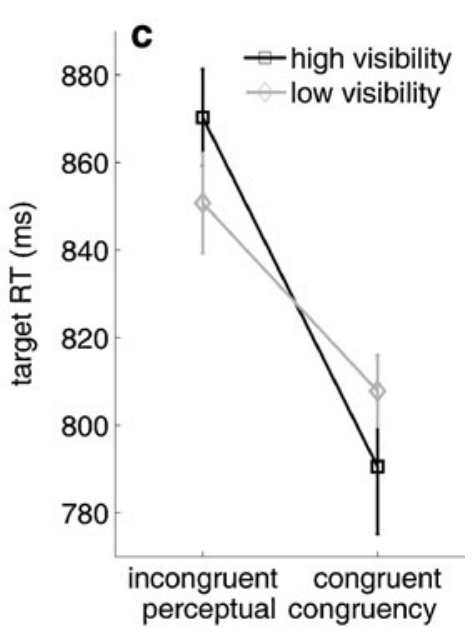

Seven participants were omitted from the analysis due to insufficient trial counts. (c) The analysis from panels $\mathbf{a}$ and $\mathbf{b}$ was repeated, this time ignoring the factor of SOA. The effect of visibility on perceptual interference is evident in the significant Perceptual Congruency $\times$ Visibility interaction $(p=.002)$. Error bars represent within-subjects standard errors (Morey, 2008)

relationship between stimulus congruency and perceptual congruency is mediated by the accuracy of flanker identification. When the flankers are correctly identified, then stimulus congruency and perceptual congruency will have the same status (actual and perceived flanker identity must be both congruent or both incongruent with the target); and when flankers are incorrectly identified, then stimulus congruency and perceptual congruency will have opposing statuses (if actual flanker identity is congruent with the target, then perceived flanker identity must be incongruent with the target, and vice versa). Thus, in the prior analyses, stimulus congruency and perceptual congruency are more correlated at short and long SOAs, where correct flanker identifications are more frequent.

In order to better discern between the effects of stimulus congruency and perceptual congruency, we performed a fully factorial analysis. In order to attain a sufficient number of trials for this analysis, it was necessary to collapse across the factor of SOA. We categorized each trial according to the conjunction of stimulus congruency, perceptual congruency, and reported flanker visibility (again, defined according to a median split). Here, we treated perceptual congruency as a graded phenomenon modulated by visibility, such that perceptual (in)congruency was considered stronger for highly visible than for poorly visible flankers. We thus performed a repeated measures ANOVA on median target RTs for correct target identifications, with a 2 (stimulus congruency) $\times 4$ (perceptual congruency: strongly incongruent, weakly incongruent, weakly congruent, or strongly congruent) design. Three participants were omitted from the analysis because they did not have at least five trials in every Stimulus Congruency $\times$ Perceptual Congruency data 
cell, leaving a total of 23 participants. The data for this analysis are plotted in Fig. 4.

This analysis allowed us to discern the independent contributions of stimulus congruency and perceptual congruency to interference with target processing. Distinctive patterns in the data should emerge that will depend on whether only stimulus congruency contributes to response interference (Fig. 4a); only perceptual congruency contributes to response interference (Fig. 4b); or both make independent, additive contributions (Fig. 4c). (The possibility of an interaction between the two types of congruency is not pictured in Fig. 4.) The pattern in the empirical data was consistent with the hypothesis that both stimulus congruency and perceptual congruency make independent, additive contributions to response interference (Fig. 4d). Indeed, the ANOVA revealed significant main effects of stimulus congruency $(p=.015)$ and perceptual congruency $(p=.003$; with a significant linear trend, $p=.003)$ and no interaction $(p>9)$. Target RTs were $31 \mathrm{~ms}$ faster for stimulus-congruent than for stimulusincongruent trials, as well as $35 \mathrm{~ms}$ faster for perceptually congruent than for perceptually incongruent trials. The latter effect grew to $45 \mathrm{~ms}$ when comparing target RTs for highly visible perceptually congruent trials with target RTs for highly visible perceptually incongruent trials. This is further evidence that the disruptive effect of flankers on target processing depends partially on the manner in which the flankers are subjectively perceived.

An alternative way of performing the prior analysis would be to consider Perceptual Congruency and Reported Flanker Visibility as independent factors. This yields a 2 (stimulus congruency) $\times 2$ (perceptual congruency) $\times 2$ (reported flanker visibility) design for the repeated measures ANOVA on target RTs. In this analysis, there were main effects of stimulus congruency ( $p=.015$ ) as well as perceptual congruency $(p=.006)$, with a marginal interaction between perceptual congruency and reported flanker visibility $(p=.1)$ due to the fact that higher flanker visibility increased target RTs under perceptual incongruence but decreased target RTs under perceptual congruence. This suggests that the contribution of reported flanker visibility to response conflict may be somewhat smaller than the contribution of reported flanker identity.

\section{RTs for flanker identification}

Because we also measured RTs for flanker identification, it could be of interest to probe response conflict effects for the flanker responses. However, this analysis is complicated by several factors. Because identification of the flankers always occurred after identification of the target, measuring RTs for the flankers with respect to flanker onset would confound the time needed to process the flanker with the time needed to process the target. An alternative is to measure flanker
RTs as the time between the target response and the flanker response. This is also not an ideal measure of flanker processing, as a substantial amount of flanker processing likely occurred during the time between stimulus onset and the target response. Thus, the present paradigm does not offer an ideal way of assessing flanker RTs. Additionally, it is not possible to assess the independent contributions of stimulus congruency and perceptual congruency on RTs for correct flanker responses, since stimulus congruency and perceptual congruency only dissociated for incorrect flanker identifications.

With these limitations in mind, we conducted an analysis on the effect of target-flanker congruency on flanker RTs (defined as the time between the target response and flanker response) using a 2 (congruency) $\times 7$ (SOA) repeated measures ANOVA. This analysis revealed a main effect of congruency $(p<.001)$, which was qualified by a Congruency $\times$ SOA interaction $(p=.012)$. The interaction arose because the effect of response conflict on flanker RTs was a Ushaped function of SOA, similar to the pattern of response conflict versus SOA for the target stimulus, as depicted in Fig. 2. This suggests that the disruption of flanker processing due to target-flanker incongruency follows a pattern similar to that of the disruption of target processing. At short and long SOAs, where the flanker is more strongly processed, incongruency between the target and the flankers is more deleterious to RTs for both the target and the flankers.

\section{Discussion}

Contributions of subjective visibility to response interference

It has previously been reported that response interference effects due to distractor stimuli can be observed even when the distractor stimuli cannot be discriminated at abovechance levels (Eimer \& Schlaghecken, 2002; Schwarz \& Mecklinger, 1995; Sumner \& Husain, 2008; Vorberg et al., 2003). On the basis of these findings, one might think that response interference does not depend on the subjective perception of the distractor stimuli, but rather is driven mainly by automatic processing of the physical properties of the distractor stimuli. We investigated this issue by presenting a suprathreshold target stimulus with flanking distractor stimuli at threshold levels of discriminability and collecting reports about target identity, flanker identity, and subjective reports of flanker visibility on every trial.

In Lau and Passingham (2006), we used a similar procedure for metacontrast-masked stimuli and showed that at certain SOAs, even when stimulus identification performance was matched, there were reliable differences in subjective visibility ratings. We further found that these differences in 
a

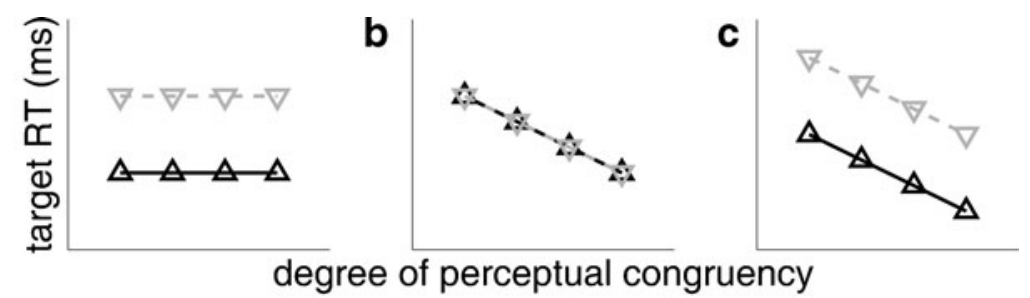

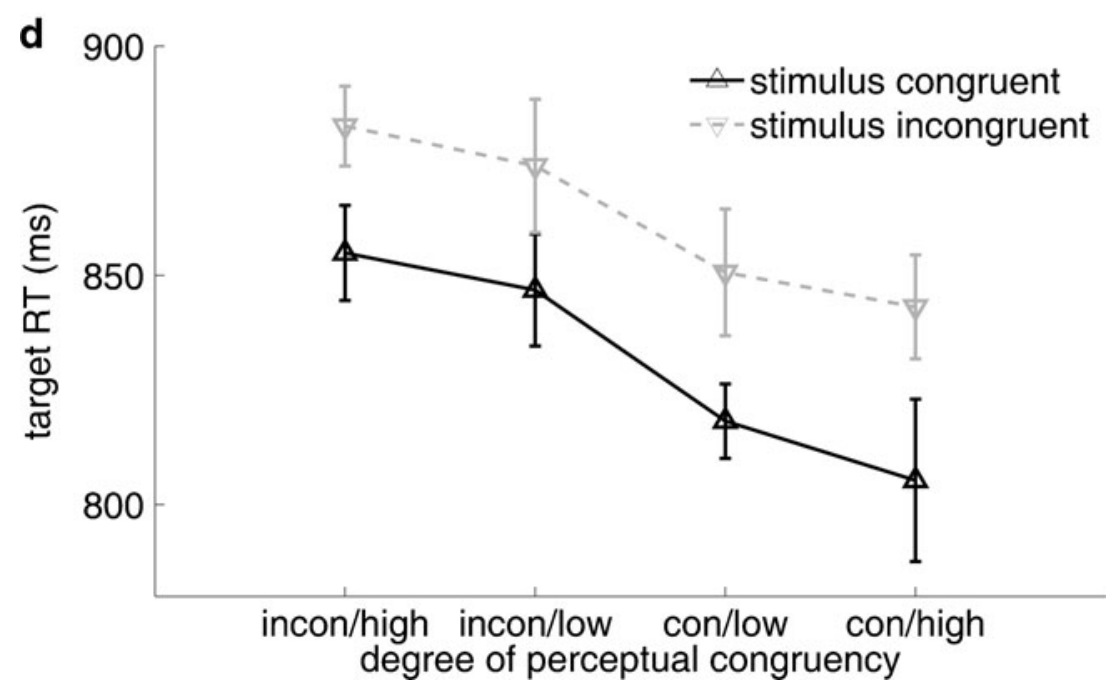

Fig. 4 Factorial analysis of stimulus and perceptual congruency. In order to better distinguish the contributions of stimulus congruency and perceptual congruency to target RTs, we investigated the independent effects of stimulus and perceptual congruency. This analysis was collapsed across SOAs in order to yield a sufficient number of trials for analysis. (a-c) Hypothetical patterns of target RTs as a function of both stimulus and perceptual congruency. These plots show expected patterns of target RTs as a function of stimulus and perceptual congruency, given different hypotheses about the relative contributions of each to interference with target RTs. Here, we combine reports about flanker identity and visibility in order to create a graded scale of perceptual congruency (on the $x$-axes, at the leftmost point flankers are perceptually incongruent and highly visible, and at the rightmost point, the flankers are perceptually congruent and highly visible). Separate lines

subjective visibility ratings were reflected by fMRI activity in the prefrontal cortex. Because response interference effects have also been observed in the prefrontal cortex, we hypothesized that subjective visibility ratings may be particularly relevant to such effects.

In the present experiment, we expected to find an acrossSOA dissociation between flanker identification performance and subjective reports of flanker visibility. A comparison of SOAs with similar flanker identification performance and dissimilar ratings of visibility would have provided a strong test of the hypothesis that the subjective visibility of the flankers drives response conflict for the target, since such a comparison would allow for inferences about the functional consequences of flanker visibility without the confounding influence of the strength of objective flanker processing (as indexed by the percentage of correct flanker responses). Unfortunately, we did not observe the expected performance-visibility indicate stimulus congruency and stimulus incongruency. (a) Effect of stimulus congruency only: Target RTs differ across stimulus congruency conditions but are insensitive to perceptual congruency. (b) Effect of perceptual congruency only: Target RTs decrease with increasing perceptual congruency but are insensitive to stimulus congruency. (c) Effects of both stimulus congruency and perceptual congruency: Target RTs are sensitive to both stimulus congruency and perceptual congruency (with no interactions). (d) Observed data: The empirical data resemble panel $\mathbf{c}$, suggesting that stimulus congruency and perceptual congruency have independent, additive effects on target RTs. A repeated measures ANOVA revealed main effects of both stimulus congruency $(p=.015)$ and perceptual congruency $(p=.003)$, with no interaction $(p>9)$. Error bars represent within-subjects standard errors (Morey, 2008)

dissociation for the flankers in these data, which necessitated using alternative means of teasing out the contribution of subjective perception to target response interference.

This does not mean that the dissociation effect is in itself unreliable, because the effect has been replicated in the fMRI scanner (Lau \& Passingham, 2006) as well as in other experiments (Maniscalco \& Lau, 2010). However, in this experiment the flanker stimuli were not presented at the focus of spatial attention, and this was perhaps one reason that the dissociation effect was not observed. This seems to suggest that the dissociation effect observed by Lau and Passingham may be sensitive to these contextual factors.

Nonetheless, the results of the present experiment suggest that response interference for a target depends on both automatic, unconscious processing of distractor stimuli and conscious perceptual processing of the stimulus. We found that defining flanker-target congruency with respect to reported 
flanker identities rather than actual flanker identities yielded a stronger overall response interference effect (Fig. 2). Furthermore, this effect of response interference due to reported flanker identity was stronger when the flankers were rated as being more subjectively visible (Figs. 3a-3c). Finally, a fully factorial analysis revealed that both the physical identity and the perceived identity and visibility of flanker stimuli make independent, additive contributions to response interference (Fig. 4). Collectively, these results demonstrate that the manner in which distractor stimuli are subjectively perceived makes an important contribution to response interference.

If this interpretation is correct, why can interference effects be observed even when distractor stimuli cannot be discriminated at above-chance levels? This apparent conundrum can perhaps be illuminated by means of the following analogy: Whereas we all agree that the function of locomotion is supported by the possession of legs, animals with their legs amputated can nonetheless move around in some limited fashion. Likewise, even if response interference is a process that essentially depends on subjective perception, perhaps it should not be surprising that when subjective perception is not informative with regard to stimulus identity, there is still some small effect of response interference. The same point is made by the observation that on a plot of response interference versus distractor visibility, interference may exist when visibility is entirely absent (i.e., the intercept may be greater than zero), even if visibility also contributes to response interference (i.e., even if the slope is greater than zero). The claims that visibility contributes to response interference and that response interference does not require visibility are thus not mutually exclusive.

Such considerations speak to the limitations of single dissociations (Schmidt \& Vorberg, 2006). Demonstrating that some factor $F$ (e.g., subjective perception) is necessary for some process $P$ (e.g., response interference) is one way to show that $F$ contributes to $P$. However, the converse is not true: Failure to show that $F$ is necessary for $P$ does not imply that $F$ does not contribute to $P$. In the present case, even if the subjective perception of a distractor stimulus may not be "necessary" for that distractor to produce target response interference, it nonetheless remains possible that subjective perception of the distractor can enhance or drive response interference in other important ways.

Levels of stimulus processing and target-distractor conflict

Of particular interest in the present analysis is the finding that the objective, physical identity of the flanker stimuli and the subjectively perceived identity of those stimuli make additive contributions to response interference (Fig. 4). This result suggests a clean separation between the processes responsible for the stimulus interference and perceptual interference effects of the flanker stimuli, as if there were two independent sources of response conflict arising from distinct levels or stages of flanker processing. This inference is justified by the observation that we would normally expect the two factors to interact, rather than to add, if they were both driven by a shared underlying set of mechanisms (Nassauer \& Halperin, 2003; Sergeant, 1996; Sternberg, 1969; Sternberg, 2001).

More precisely, following the logic of Sternberg (2001), the results suggest the existence of two processing operations arranged in serial (in the sense that the operation of one must complete before the operation of the next commences) that are independent (in the sense that the operation of each can be varied while holding the other constant). The processing stage corresponding to stimulus interference would require high-fidelity representations of the target and flanker stimuli, even at SOAs at which perception of the flankers was relatively poor. This observation is suggestive of the possibility that stimulus interference may originate at early stages of processing that are temporally prior to, and/or neurologically upstream from, the spatiotemporal locus of neural processing within which the disruptive effects of metacontrast masking on flanker processing occur. The processing stage corresponding to perceptual interference is presumably a later, perceptual or postperceptual, stage of processing that occurs during or after the formation of the conscious percepts of the target and flankers. Thus, the present data seem particularly amenable to theories of consciousness that emphasize a functionally and anatomically hierarchical relationship between early, posterior, unconscious processing and late, anterior, conscious processing (e.g., Dehaene \& Naccache, 2001; Lau \& Rosenthal, 2011).

Although it seems likely that the response interference due to reported flanker identity occurs at a relatively high level of processing, it is not likely to originate at the level of compatibility of motor outputs used to make identification responses for targets and flankers; participants used the left hand to provide target identification responses and the right hand to provide flanker identification responses. Furthermore, the finger-response mappings were reversed for the two hands: For the left hand, the index finger was used to indicate a "diamond" response and the middle finger was used to indicate "square," whereas for the right hand, the index finger indicated "square" and the middle finger indicated "diamond."

It is also an a priori possibility that the response interference due to reported flanker identity is not associated with perception of the flankers per se, but rather is driven by the activation of the semantic content associated with the flanker identification response. For instance, it is possible that even on trials on which the flankers are not perceived at all, the response content of a participant's guess about the identity of the flankers might interfere with the response content generated by target processing. However, this possibility seems unlikely. In this experiment, the flanker identification response was made after the target identification response, so a guess about flanker identity could not affect the target 
RT unless the flanker guess was made (unnecessarily) prior to the target response. Additionally, the interfering effect associated with reported flanker identity was stronger when the flankers were reported to be more subjectively visible (Figs. 3 and 4), which is consistent with the interpretation that response interference associated with reported flanker identity is driven directly by the perception of the flankers. It therefore seems more likely that response interference was driven by the perception of the flankers at the time of stimulus presentation, rather than by the semantic content of the perceptual decision made about the flankers.

Alternative interpretations of the perceptual interference effect

Although we have taken these results as indicating the effects of flanker perception on target RTs, an alternative interpretation of the data would be that the causality flows in the opposite direction, such that reports of flanker identity and the level of flanker visibility are influenced by variations in target RTs. For instance, it could be that participants are sensitive to variations in their target RTs and attribute shorter target RTs to the presence of congruent flankers, thereby producing a corresponding bias in their reports on flanker identity and visibility. Such a phenomenon would be consistent with the data in Fig. 4d, in which shorter target RTs are associated with higher degrees of perceptual congruency.

We can gain empirical traction for this alternative explanation if we suppose that the hypothesized biasing effect of target RTs on flanker responses does not depend on the flanker-mask SOA. Granted this assumption, we should expect to find that those SOAs with shorter target RTs are associated with higher probabilities of reporting the flankers to be congruent with the target. That is, across SOAs, we should find a negative correlation between target RTs and the probability of perceptual congruency.

However, if anything, the correlation in the data runs in the opposite direction. When data are averaged across all participants before running the correlation, the correlation between target RT and the probability of perceptual congruency across the seven SOA data points is strongly positive (Pearson's $r=.86, p=.014$; Spearman's $\rho=.43, p=.3$ ). When considering the across-SOA correlation for each participant individually, the mean correlation coefficient is smaller as a result of substantial between-subjects variation, but still positive rather than negative (mean Pearson's $r=$ .08 , mean Spearman's $\rho=.07$ ). Note that these patterns are not problematic for the account that perceptual congruency influences target RTs, because this account is not committed to specifying any particular relationship between overall flanker response profiles and the overall target RT; this account only applies to the differences between target RTs that are conditional on perceptual congruency. Thus, the relationship between target RTs and perceptual congruency exhibited by the present data seems unlikely to be due to a simple biasing effect of the overall target RT on flanker responses.

\section{Limitations and future directions}

One important caveat for the present study is that, because the task design required participants to respond to both the central target and the flanking distractor stimuli on every trial, the distractor stimuli likely received more attention than they would have, had there been no requirement to identify the distractors, as is typical in response conflict studies utilizing distractor stimuli. We believe that this issue is attenuated to some extent by the fact that the target stimulus required a speeded response with tight time pressure (participants were instructed to respond to the target as quickly and accurately as possible, and target identifications had to be given within $2 \mathrm{~s}$ or else the trial was terminated), whereas flanker responses were unspeeded (participants were instructed that the RT for flankers was not important, and they were given up to $5 \mathrm{~s}$ after entry of the target identification to provide the flanker identification and visibility rating). Additionally, the target response always was given prior to the flanker responses, and the target stimulus was presented foveally at a suprathreshold contrast, whereas the flankers were presented peripherally at threshold levels of discriminability. For all of these reasons, it is likely that the target stimulus was more perceptually and cognitively salient, and thus received more attention, than the flankers.

Nonetheless, we cannot rule out the possibility that the conclusions of the present study are limited to cases in which some attentional resources are allocated to the distractor stimuli. Future studies using modified behavioral designs, or using neuroscience methods to indirectly probe distractor processing, will be needed to shed light on this issue.

\section{Conclusion}

Because response interference can occur even when perceptual identification of distractor stimuli is at chance levels, it may seem that perception of distractor stimuli is superfluous to response interference and that automatic processing of physical stimulus identity is sufficient to explain response interference. However, the current research suggests that while perception of distracters may not be necessary to trigger response interference, the manner in which distractors are subjectively perceived can nonetheless contribute to response interference. Specifically, actual and perceived target-distractor incongruency can make contributions to target response interference that are similar in magnitude and functionally independent. 
Author note This work was supported by internal funding from Columbia University (to H.L.) and the Templeton Foundation (Grant 15462-SCI04). F.C.-F. is a "La Caixa" Foundation fellow.

\section{References}

Botvinick, M. M., Cohen, J. D., \& Carter, C. S. (2004). Conflict monitoring and anterior cingulate cortex: An update. Trends in Cognitive Sciences, 8, 539-546. doi:10.1016/j.tics.2004.10.003

Brainard, D. H. (1997). The Psychophysics Toolbox. Spatial Vision, 10, 433-436. doi:10.1163/156856897X00357

Breitmeyer, B. G., \& Öğmen, H. (2006). Visual masking: Time slices through conscious and unconscious vision (2nd ed.). Oxford, U.K.: Oxford University Press.

Dehaene, S., \& Naccache, L. (2001). Towards a cognitive neuroscience of consciousness: basic evidence and a workspace framework. Cognition, 79, 1-37. doi:10.1016/S0010-0277(00)00123-2

Eimer, M., \& Schlaghecken, F. (2002). Links between conscious awareness and response inhibition: Evidence from masked priming. Psychonomic Bulletin \& Review, 9, 514-520. doi:10.3758/BF03196307

Eimer, M., \& Schlaghecken, F. (2003). Response facilitation and inhibition in subliminal priming. Biological Psychology, 64, 726. doi:10.1016/S0301-0511(03)00100-5

Eriksen, C. W., \& Schultz, D. W. (1979). Information processing in visual search: A continuous flow conception and experimental results. Perception \& Psychophysics, 25, 249-263. doi:10.3758/ BF03198804

Kouider, S., \& Dehaene, S. (2007). Levels of processing during nonconscious perception: A critical review of visual masking. Philosophical Transactions of the Royal Society B, 362, 857875. doi:10.1098/rstb.2007.2093

Lau, H. (2009). Volition and the functions of consciousness. In M. S. Gazzaniga (Ed.), The cognitive neurosciences (4th ed., pp. 11911200). Cambridge, MA: MIT Press, Bradford Books.

Lau, H. C., \& Passingham, R. E. (2006). Relative blindsight in normal observers and the neural correlate of visual consciousness. Proceedings of the National Academy of Sciences, 103, 1876318768. doi:10.1073/pnas.0607716103

Lau, H., \& Rosenthal, D. (2011). Empirical support for higher-order theories of conscious awareness. Trends in Cognitive Sciences, 15, 365-373. doi:10.1016/j.tics.2011.05.009

Macmillan, N. A., \& Creelman, C. D. (2005). Detection theory: A user's guide (2nd ed.). Mahwah, NJ: Erlbaum.

Maniscalco, B., \& Lau, H. (2010). Comparing signal detection models of perceptual decision confidence. Naples, FL: Paper presented at the meeting of the Vision Sciences Society.

Merikle, P. M., Joordens, S., \& Stolz, J. A. (1995). Measuring the relative magnitude of unconscious influences. Consciousness and Cognition, 4, 422-439. doi:10.1006/ccog.1995.1049
Morey, R. D. (2008). Confidence intervals from normalized data: A correction to Cousineau (2005). Tutorials in Quantitative Methods for Psychology, 4, 61-64.

Morsella, E., Gray, J. R., Krieger, S. C., \& Bargh, J. A. (2009). The essence of conscious conflict: Subjective effects of sustaining incompatible intentions. Emotion, 9, 717-728.

Nassauer, K. W., \& Halperin, J. M. (2003). Dissociation of perceptual and motor inhibition processes through the use of novel computerized conflict tasks. Journal of the International Neuropsychological Society, 9, 25-30.

Neumann, O., \& Klotz, W. (1994). Motor responses to nonreportable, masked stimuli: Where is the limit of direct parameter specification? In J. Umiltà \& M. Moscovitch (Eds.), Attention and performance $X V$ : Conscious and nonconscious information processing (pp. 123-150). Cambridge, MA: MIT Press.

Pelli, D. G. (1997). The VideoToolbox software for visual psychophysics: Transforming numbers into movies. Spatial Vision, 10, 437442. doi: $10.1163 / 156856897 X 00366$

Schmidt, T., \& Vorberg, D. (2006). Criteria for unconscious cognition: Three types of dissociation. Perception \& Psychophysics, 68, 489-504. doi:10.3758/BF03193692

Schwarz, W., \& Mecklinger, A. (1995). Relationship between flanker identifiability and compatibility effect. Perception \& Psychophysics, 57, 1045-1052. doi:10.3758/BF03205463

Sergeant, J. (1996). A theory of attention: An information processing perspective. In G. R. Lyon \& N. A. Krasnegor (Eds.), Attention, memory, and executive function (pp. 57-69). Baltimore, MD: Paul H. Brookes.

Sternberg, S. (1969). The discovery of processing stages: Extensions of Donders' method. Acta Psychologica, 30, 276-315. doi:10.1016/ 0001-6918(69)90055-9

Sternberg, S. (2001). Separate modifiability, mental modules, and the use of pure and composite measures to reveal them. Acta Psychologica, 106, 147-246. doi:10.1016/S0001-6918(00)00045-7

Stins, J. F., Polderman, J. C. T., Boomsma, D. I., \& de Geus, E. J. C. (2007). Conditional accuracy in response interference tasks: Evidence from the Eriksen flanker task and the spatial conflict task. Advances in Cognitive Psychology/University of Finance and Management in Warsaw, 3, 409-417.

Sumner, P., \& Husain, M. (2008). At the edge of consciousness: Automatic motor activation and voluntary control. Neuroscientist, 14, 474- 486.

Van den Bussche, E., Van den Noortgate, W., \& Reynvoet, B. (2009). Mechanisms of masked priming: A meta-analysis. Psychological Bulletin, 135, 452-477. doi:10.1037/a0015329

Vorberg, D., Mattler, U., Heinecke, A., Schmidt, T., \& Schwarzbach, J. (2003). Different time courses for visual perception and action priming. Proceedings of the National Academy of Sciences of the United States of America, 100, 6275-6280.

Watson, A. B., \& Pelli, D. G. (1983). QUEST: A Bayesian adaptive psychometric method. Perception \& Psychophysics, 33, 113-120. doi:10.3758/BF03202828 